\title{
Anneau vaginal à la progesterone: Appel a l'action pour combler les ecarts importants dans la planification familiale postnatal
}

Population Council

Follow this and additional works at: https://knowledgecommons.popcouncil.org/departments_sbsr-rh

Part of the Demography, Population, and Ecology Commons, Family, Life Course, and Society Commons, International Public Health Commons, and the Women's Health Commons How does access to this work benefit you? Let us know!

\section{Recommended Citation}

"Anneau vaginal à la progesterone: Appel a l'action pour combler les ecarts importants dans la planification familiale postnatal," Fiche de promotion. New York: Population Council, 2015. 


\section{ANNEAU VAGINAL A LA PROGESTERONE \\ APPEL A L'ACTION POUR COMBLER LES ECARTS IMPORTANTS DANS LA PLANIFICATION FAMILIALE POSTNATAL}

L'anneau vaginal à la progestérone (AVP) est une méthode efficace et sûre de contraception pour les femmes qui allaitent. C'est une médecine prioritaire de la liste des produits médicaux essentiels de l'OMS et elle est inclue parmi les critères médicaux éligibles pour les personnels de la planification familiale.

Des grossesses rapprochées peuvent avoir de sérieuses implications de santé pour la mère et le nouveau-né et sont une des causes principales de mortalité pour les mères et les enfants. Pourtant plus de 65\% des femmes en situation

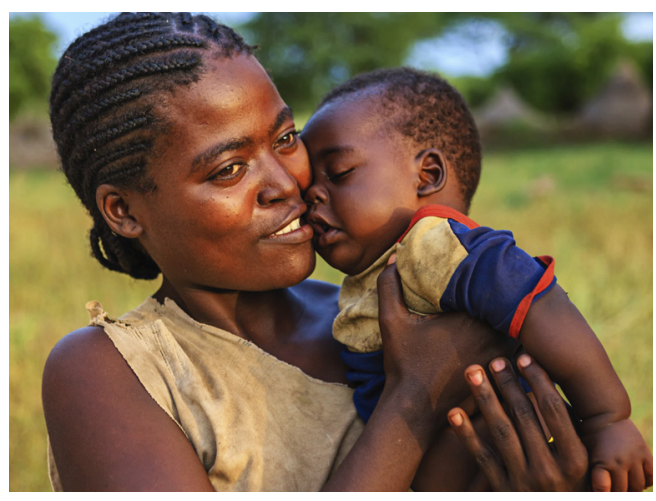
postnatale disent ne pas utiliser de méthode contraceptive dans l'année suivant l'accouchement. Développé par le Population Council, I'AVP est un anneau intra-vaginal qui donne une option contraceptive aux femmes qui allaitent au moins quatre fois par jour dès la quatrième semaine après l'accouchement. II peut être utilisé sans problème au cours de la première année suivant l'accouchement afin d'espacer ou d'empêcher de futures grossesses.

\section{FAIRE CONNAITRE L'AVP DANS LES PAYS EN VOIE DE DEVELOPPEMENT POURRAIT ACCROITRE LE CHOIX, L'ACCESSIBILITE ET LA DISPONIBILITE DES METHODES ET OPTIONS CONTRACEPTIVES POUR LES FEMMES EN SITUATION POSTNATALE.}

\section{ACCROITRE LE CHOIX DE LA METHOD}

L'AVP ne nécessite pas d'action quotidienne (à l'inverse de la pilule) et est une alternative efficace pour les femmes qui ne souhaitent pas utiliser de méthodes cliniques (tels que les moyens intra-utérins ou les implants) ou les méthodes avec barrières (contraceptifs masculins ou féminins).

\section{Des rapports d'études} cliniques indiquent que pas le plaisir sexuel, et que la plupart des partenaires ne sentent pas l'anneau durant le rapport. l'utilisation de l'AVP n'affecte

\section{AMELIORE LA SANTE DE LA MERE ET DE L'ENFANT \\ ACCROIT L'ACCESSIBILITE DE LA METHODE}

L'AVP aide les femmes en situation postnatale à accroitre l'intervalle entre les naissances et empêche les grossesses non planifiées, en protégeant leur santé. L'AVP pouvant être utilisé pendant l'allaitement et le lait maternel augmentant l'immunité, il contribue à une nutrition positive de l'enfant et à une meilleure santé. Pour que l'AVP soit efficace les femmes doivent allaiter leurs enfants au moins 4 fois par jour. Cependant, au contraire des femmes utilisant la méthode d'allaitement maternel et d'aménorrhée pour l'espacement des naissances, elles peuvent aussi donner d'autres aliments à leur enfant.
Le manque de personnel de santé formé est un défi important pour le service offert par la planification familiale. En tant que méthode d'utilisateur averti, I'AVP requiert une interaction minimum entre l'utilisateur et le personnel de santé. Le personnel de santé a besoin de formation pour conseiller les femmes sur la manière d'utiliser et d'enlever l'anneau. En suivant le «cadre d'optimisation du personnel de santé » de l'OMS, le partage des tâches par lequel les anneaux sont distribués par les sagesfemmes, les infirmières, la communauté des personnels de santé, et les pharmaciens, peut aider à accroitre l'accessibilité de l'AVP.
ACCROIT LA

DISPONIBILITE DE LA METHODE

Comme il est facile à utiliser, I'AVP peut être distribué dans les secteurs public, privé et à but non lucratif: dans les cliniques, les points de vente de communautés, pharmacies, et les franchises sociales. Il peut aussi être intégré aux services de planification familiale et de santé maternelle et infantile. Cette disponibilité au travers de l'ensemble du marché permettra aux personnels de santé d'atteindre tous les clients potentiels, depuis les personnes nécessitant des tarifs subventionnés jusqu'à ceux pouvant payer le prix du marché pour le produit.

POPULATION COUNCIL Ideés. Preuve. Impact. 


\section{Appel à l'action pour faire connaitre l'AVP Conformément aux recommandations de la commission de l'ONU sur les produits indispensables aux femmes et aux enfants}

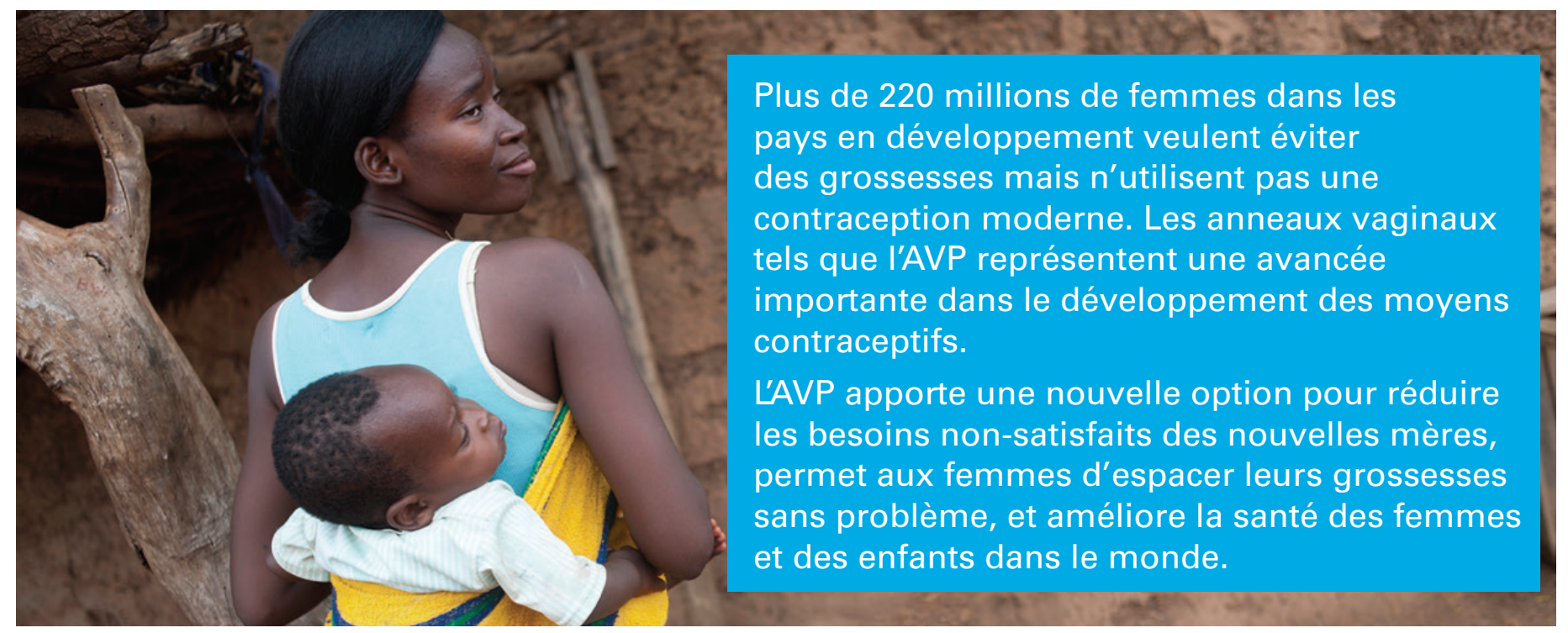

POLITIQUE NATIONALE ET AUTORITES DE REGULATION

- Enregistrer l'AVP enregistré comme un produit médical essentiel pour la planification familiale au sein des listes nationales de produits médicaux essentiels.

- Intégrer l'AVP aux formations (pendant la formation et sur le tas) pour les personnels du planification familiale pour en faire une idée dominante dans les programmes de santé, et les divers segments du marché.

- Garantir une ligne budgétaire dans le budget annuel du ministère de la santé pour fournir et distribuer l'AVP.

\section{FOURNISSEURS ET MANAGERS DE LA CHAINE LOGISTIQUE}

- Travailler avec les acheteurs mondiaux ou centralisés sur les commandes, les prix, l'approvisionnement et la distribution de l'AVP.

- Garantir un approvisionnement cohérent de l'AVP entre le public, les ONG, et les réseaux de distribution de service privé.
FOURNISSEURS DE LIVRAISON DE SERVICES

- Offrir conseil et services sur l'AVP au travers de services de santé intégrés pour la mère et l'enfant (durant la grossesse, l'accouchement, et les visites d'immunisation et de nutrition des nouveau-nés aux organisations de santé et des communautés).

- Garantir une formation sur les conseils et services sur l'AVP à tous les niveaux des personnels de santé impliqués dans la distribution de service de planification familiale, y compris les médecins, les infirmières, les sages-femmes, les personnels d'aide sociale de communauté, et les pharmaciens.

\section{GROUPES DE CLIENT ET DE PROMOTION}

- Promouvoir l'AVP auprès de clients ciblés, ex. médecins et associations d'obstétrique/gynécologie, groupes de médecins, personnels et associations infirmiers et sages-femmes, et pharmaciens.

- Utiliser les ressources des communautés pour fournir un accès aux services AVP et diffuser le matériel pédagogique sur l'utilisation et les bénéfices de I'AVP pour les femmes qui allaitent.
DONNEURS, ORGANISATTIONS DE LA SOCIETE CIVILE ET

\section{MULTILATERALES}

- Soutenir les droits des femmes en facilitant leur choix contraceptif.

- Promouvoir la compréhension du rôle de l'AVP en promouvant l'allaitement et améliorant la santé des mères et des enfants.

- Investir et soutenir la distribution de service durable pour l'AVP et fournir des subventions pour les groupes aux revenus faibles.

- Collaborer avec les agences d'assistance technique pour rassembler les données en vue d'une meilleure prévision et de stratégies de programmes de distribution pour l'AVP au sein du public et du secteur privé.

Le Population Council a développé les trois formes les plus efficaces de contraception réversibles et à effet durable: le T IUD Copper, le système intra-utérin Mirena ${ }^{\circledR}$, et l'implant contraceptif (Norplant ${ }^{\oplus}$ and Jadelle ${ }^{\circledR}$ ). 170 millions de femmes utilisent actuellement des technologies contraceptives développées par le Council.

Le Population Council trace aussi le chemin vers l'introduction de nouvelles technologies d'anneau vaginal développés par le Council, faciles à utiliser et sous le contrôle de la femme. 